\title{
Modeling of operating modes of relay protection in electrical networks with insulated neutral
}

\author{
$A$. Aluynov ${ }^{1, *}, O$. Vyatkina $^{1}, E$. Gracheva ${ }^{2}$, and $A$. Nemirovskiy ${ }^{1}$ \\ ${ }^{1}$ Vologda State University, Lenina Street 15, Vologda, 160000, Russia \\ ${ }^{2}$ Kazan State Power Engineering University, Kazan, Russian Federation
}

\begin{abstract}
This work aims to study relay protection and automation in insulated neutral networks to identify additional criteria for choosing the type of earthing. To calculate the single-phase earth fault currents as a function of the operating modes of the electrical substation, the $10 \mathrm{kV}$ network of the electrical substation was simulated in the software package. The advantages and disadvantages of centralized and non-centralized protections are shown. The paper presents the sequence of actions necessary for the correct operation of directional zero sequence protection.
\end{abstract}

\section{Introduction}

Currently, an in-depth question on the earthing of the network neutral arises at the stage of electrical project design during the construction of new, as well as the reconstruction of the existing medium voltage substations [1-4]. This problem consists in the fact that there are no clear instructions for solving this problem in the electrical installation rules. The $2-35 \mathrm{kV}$ electrical networks can be provided both with an insulated neutral and with a neutral earthed through an arc suppression reactor or a resistor [5-7]. The aim of this work is to study relay protection and automation in networks with insulated neutral, to identify additional criteria for choosing the type of earthing.

The object of research is the $10 \mathrm{kV}$ electrical network of the $110 / 35 / 10 \mathrm{kV}$ substation. The $110 \mathrm{kV}$ switchgear (SG) is formed according to the scheme "Two blocks with switches and a non-automatic repair jumper on the side of the lines"; the $35 \mathrm{kV} \mathrm{SG}$ is "One working section of busbars sectioned by a switch"; the $10 \mathrm{kV}$ SG is "One bus system sectioned by a circuit breaker".

The servicing of this substation assumes the absence of permanent operating personnel. It includes two power transformers with capacities of $10 \mathrm{MV} \bullet \mathrm{A}$, with a connection group $\mathrm{Y} 0 / \mathrm{Y} 0 / \Delta-0-11, \mathrm{UV}=115 \mathrm{kV} 9 \mathrm{x} 1.78 \%$. In a $10 \mathrm{kV}$ network, consumers have reliability categories 2 and 3.

The $10 \mathrm{kV}$ switchgear consists of two input units; sectional switch unit; sectional disconnector unit; two units with voltage transformers; two units with auxiliary transformers; 11 units of outgoing lines with switches.

Reserve lines are not considered in this study.

The parameters of $10 \mathrm{kV}$ lines required for the study are presented in Table 1.
Table 1. Data on connections for the $10 \mathrm{kV}$ switchgear.

\begin{tabular}{|c|c|c|c|}
\hline $\begin{array}{c}\text { No. of } \\
\text { unit }\end{array}$ & Connection & $\begin{array}{c}\text { Length, } \\
\mathrm{km}\end{array}$ & $\begin{array}{c}\text { Capacitive } \\
\text { current, } \\
\mathrm{A} / \mathrm{km}\end{array}$ \\
\hline \multirow{2}{*}{2} & Feeder -1 & 4.67 & 1.3 \\
\hline & Feeder -2 & 3.11 & 1.23 \\
\cline { 2 - 4 } & RP-1052, unit 3 & 1.2 & 0.9 \\
\cline { 2 - 4 } & RP-1052, unit 4 & 0.6 & 0.9 \\
\hline 3 & Feeder -3 & (Reserve) & 1.23 \\
\hline \multirow{2}{*}{4} & Feeder -4 & 2 & 1.23 \\
\cline { 2 - 4 } & RP-3036, unit 1 & 0.6 & 0.9 \\
\hline 5 & Feeder -5 & 2.56 & 1.23 \\
\hline 6 & Feeder -6 & 3.1 & 1.23 \\
\hline 7 & Feeder -7 & 2.77 & 1.3 \\
\hline \multirow{3}{*}{8} & Feeder -8 & 3.11 & 1.23 \\
\cline { 2 - 4 } & RP-1052, unit 1 & 0.4 & 0.9 \\
\cline { 2 - 4 } & RP-1052, unit 2 & 0.9 & 1.23 \\
\hline 9 & Feeder -9 & (Reserve) & 1.23 \\
\hline \multirow{2}{*}{10} & Feeder -10 & 2 & 1.23 \\
\cline { 2 - 4 } & RP-3036, unit 2 & 1 & 1.23 \\
\hline 11 & Feeder -11 & (Reserve) & 1.3 \\
\hline
\end{tabular}

\section{Materials and methods}

To calculate the single-phase earth fault currents as a function of the substation operation modes, the $10 \mathrm{kV}$ substation network was simulated in the EnergyTKZ software package. This program was developed to perform electrical calculations during design and operation of power systems of any complexity. The software complex can be used to calculate the capacitive currents of single-phase earth faults in networks with insulated neutral [8-9].

This model makes it possible to quickly calculate the capacitive current in the network during normal operation, automatic load transfer, and intentional disconnections of the connections.

\footnotetext{
* Corresponding author: alyunov@mail.ru
} 


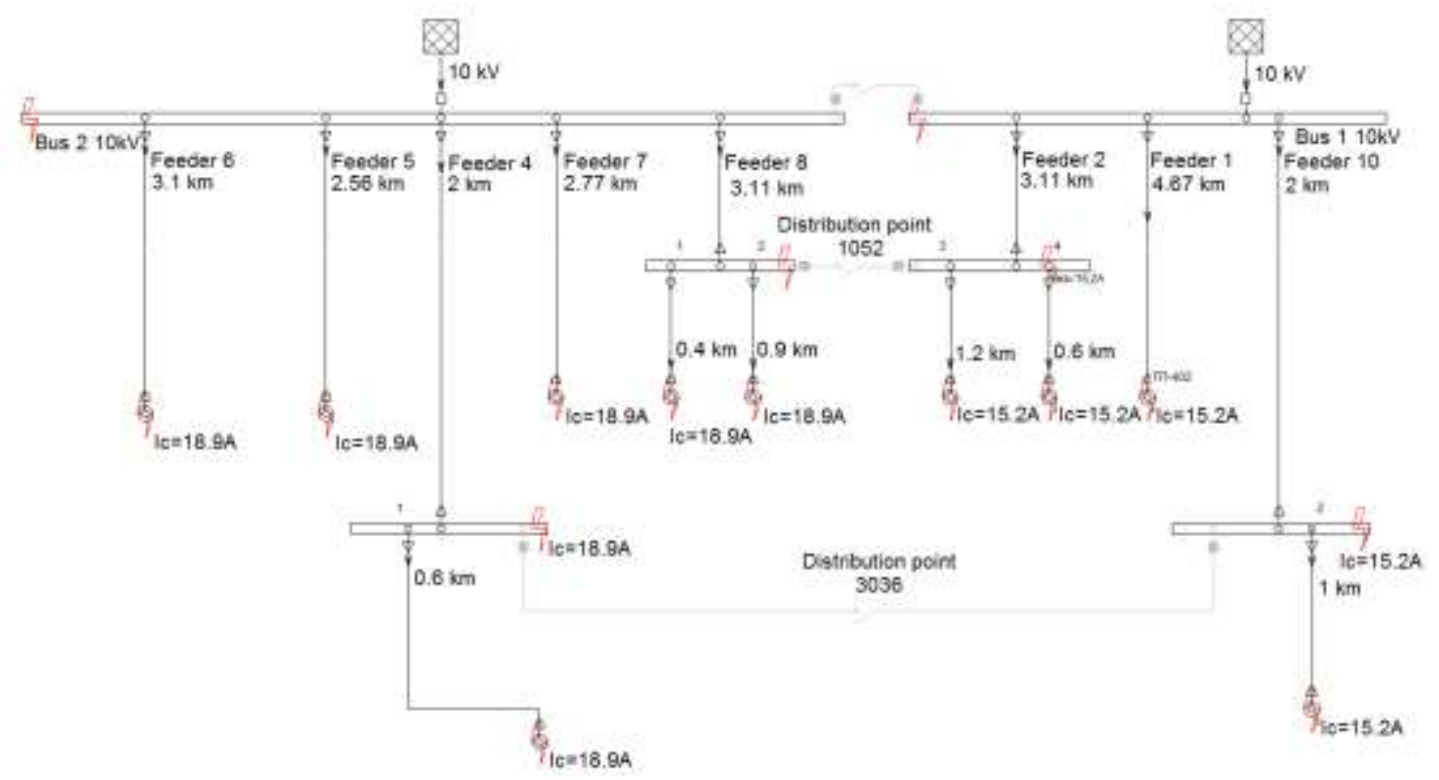

Fig. 1. Total capacitive current in the $10 \mathrm{kV}$ network during normal operation.

The $10 \mathrm{kV}$ network simulated in the EnergyTKZ software package is shown in Figure 1.

The maximum single-phase earth fault current occurs when the network gets the maximum total length of connections. This mode is typical for the post-emergency mode of the substation, that is, when the automatic transfer switch is triggered or the sectional switch is deliberately turned on by the operating personnel [10].

To calculate the operating current of non-directional zero-sequence protections, it is necessary to tune away from the maximum own capacitive currents of the connection. To determine this current, one connection at a time is switched on in the EnergyTKZ software. If the connection is an input to a distribution point, then to determine the maximum own capacitive current, it is necessary to turn on the section switch of the distribution point. The scheme to determine the maximum capacitive current on the example of Feeder-2 is shown in Figure 2.

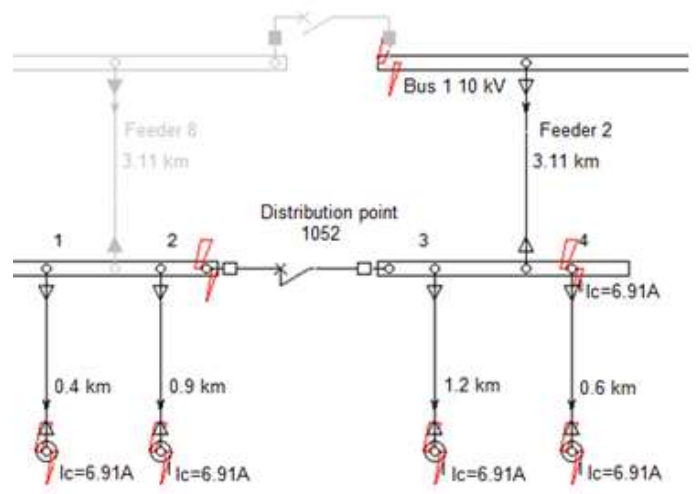

Fig. 2. The scheme to determine the maximum capacitive current on the example of Feeder-2.

The capacitive currents of the $10 \mathrm{kV}$ network with an insulated neutral are given in Table 2.
Table 2. Capacitive currents of the $10 \mathrm{kV}$ network.

\begin{tabular}{|c|c|c|c|}
\hline $\begin{array}{c}\text { No. } \\
\text { of } \\
\text { unit }\end{array}$ & Connection & $\begin{array}{c}\text { Total capacitive current } \\
\text { in the network during } \\
\text { normal operation, A }\end{array}$ & $\begin{array}{c}\text { Maximum } \\
\text { capacitive } \\
\text { current, A }\end{array}$ \\
\hline 1 & Feeder -1 & 15.2 & 6.07 \\
\hline 2 & Feeder -2 & 15.2 & 6.91 \\
\hline 4 & Feeder -4 & 18.9 & 4.23 \\
\hline 5 & Feeder -5 & 18.9 & 3.15 \\
\hline 6 & Feeder -6 & 18.9 & 3.81 \\
\hline 7 & Feeder -7 & 18.9 & 3.6 \\
\hline 8 & Feeder -8 & 18.9 & 6.91 \\
\hline 10 & Feeder -10 & 15.2 & 4.23 \\
\hline
\end{tabular}

To have an opportunity to form protection against single-phase earth faults, it is necessary to install zerosequence current transformers.

In this $10 \mathrm{kV}$ network consumers of the second reliability category have the ability to switch to a reserve independent power source, and the rest have the third category. So, it is advisable to consider protection against single-phase earth faults both with an action on a signal and on a disconnection. The structure of the designed protection against single-phase earth faults in a network with an insulated neutral is shown in Figure 3 [11].

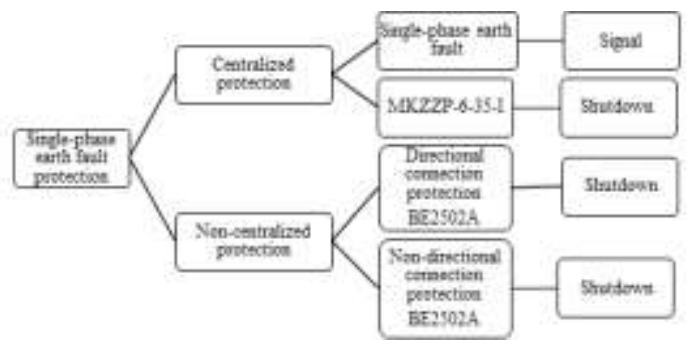

Fig. 3. Total capacitive current in the $10 \mathrm{kV}$ network during normal operation. 
Centralized protection of Sirius-OZZ. To record the presence of earthing in the $10 \mathrm{kV}$ network and determine the damaged connection, it is planned to install the Sirius-OZZ microprocessor terminal of (JSC "Radius Avtomatika"). To save space in the general substation control room and to minimize the length of the control cables, it is advisable to place the device in a sectional disconnector unit.

For its functioning, the control cables are connected to the microprocessor terminal from the voltage transformer windings, connected in an "open delta", and from the zero sequence current transformers of all connections.

The AC circuits of the device are connected to zero sequence current transformers of all connections, including reserve lines. The terminal settings have a function of usage the current channels. This setting allows the device to understand whether the current channel is connected to the residual current transformer and whether it is necessary to query this channel. Thus, the current inputs to which the residual current transformers are connected will not be queried. If it becomes necessary to put these lines into operation, the corresponding current channels can be queried in the terminal settings [12].

Support for the IEC61850 protocol facilitates the use of the Sirius-OZZ terminal in the concept of digital substations. This project provides for the organization of a communication line with the Modbus TCP exchange protocol.

To determine the presence of single-phase earth fault current in the network, the device controls the value of $3 \mathrm{U} 0$. The recommended setpoint is $25-30 \mathrm{~V}$, taking into account the worst case scenario. The detuning time from short-term surges of $3 \mathrm{U} 0$, as a rule, is taken as $9 \mathrm{~s}$.

An important feature of the centralized single-phase earth fault protection is the event recorder. The device stores in memory the facts of detecting malfunctions with reference to astronomical time, thereby realizing an archive of events. In this case, any device start-up, arrival of a discrete signal, detection of an internal malfunction is recorded in the event memory with the assignment of the date and time of the moment of detection. The ability to record the presence of faults in the event archive is a very useful function for a substation without permanent personnel on duty [10].

The Sirius-OZZ device is considered to be quite effective if there are a sufficient number of connections in the uncompensated neutral network. For example, if on the first section of the busbars of the $10 \mathrm{kV}$ network of the considered substation one of the three bays is disconnected, the non-directional protection of relative metering will not be able to operate selectively, the device will simply inform about the presence of earthing in the network.

The centralized protection MKZZP-6-35-I. As it is known, the presence of a single-phase earth fault is characterized by a large number of negative factors. Therefore, if in a network with an insulated neutral, a sudden disconnection of an element does not lead to a disruption in the power supply and the technological process for consumers, then the action of protection against single-phase earth faults on disconnection can be considered.

The centralized protection MKZZP-6-35-I most often is used in cases where the existing protection and automation of connections has already been formed on electromechanical relays, since almost all microprocessor-based feeder protection terminals have an additional function of protection against single-phase earth faults.

The protection of this device by its operation principle is a centralized current non-directional with relative measurement. The fact of the presence of a single-phase earth fault is also recorded in the central alarm terminal by activating the "Protection trip" relay. Protection is performed with definite time delay. At zero value of the setpoint, the minimum response time of the protection is $40 \mathrm{~ms}$ [11,12].

The head protection device has two RS-485 interfaces, which perform connection with the APCS and connect a computer without removing the operational power supply of the device.

Consider an example in which, on the $10 \mathrm{kV}$ side of the first section of substation busbars, one of the connections (for example, Feeder-2) for technical reasons was taken out for repair. In the event of an earth fault at the Feeder 1 connection, a single-phase earth fault current is generated, which is specific to the capacitive current of the undamaged Feeder-10 feeder. The earth fault current passing through the two connections will be exactly the same, the difference will be only in the flow direction. Thus, the device, reading the analog signals, comes to the conclusion that the total currents on the two connections is the same. Therefore, the protection action will be insulated only by a signal about the presence of earthing in the network.

During the study of this type of protection, it turned out that the principle of fixing a connection with damage by identification the highest single-phase earth fault current of the connection is operable only in a network with a large number of connections. However, if the network consists of feeders with cable and overhead lines, a large difference in their capacitive currents will not affect the efficiency (selectivity) of protection, since relative metering does not require tuning out from its own capacitive current.

Non-centralized protection BE2502A0103. This microprocessor terminal has both directional and nondirectional single-phase earth fault protection.

The $10 \mathrm{kV}$ connection protection and automation devices are installed in the relay compartment of the corresponding connection units. To begin with, it is necessary to consider protection against single-phase non-directional earth faults, since it is much easier in terms of implementation. Also, the determination of the operation current of the protection of non-directional protection is more reliable than that of directional protection.

To ensure functioning of the non-directional zero sequence protection, an analog signal $3 \mathrm{I} 0$ must be connected to the bay terminal from the zero sequence current transformers located in the feeder units. 
Table 3. Calculation results of non-directional zero sequence protection settings.

\begin{tabular}{|c|c|c|c|c|}
\hline $\begin{array}{c}\text { No. } \\
\text { of } \\
\text { unit }\end{array}$ & Connection & $\begin{array}{c}\text { Protection } \\
\text { operation } \\
\text { current, A }\end{array}$ & $\begin{array}{c}\text { Relay } \\
\text { operation } \\
\text { current, A }\end{array}$ & $\begin{array}{c}\text { Sensitivity } \\
\text { coefficient }\end{array}$ \\
\hline 1 & Feeder-1 & 7.28 & 0.12 & 1.25 \\
\hline 2 & Feeder-2 & 8.29 & 0.14 & 1 \\
\hline 4 & Feeder-4 & 5.08 & 0.08 & 1.85 \\
\hline 5 & Feeder-5 & 3.78 & 0.06 & 2.76 \\
\hline 6 & Feeder-6 & 4.57 & 0.08 & 2.14 \\
\hline 7 & Feeder-7 & 4.32 & 0.07 & 2.31 \\
\hline 8 & Feeder-8 & 8.29 & 0.14 & 0.98 \\
\hline 10 & Feeder-10 & 5.08 & 0.08 & 2.16 \\
\hline
\end{tabular}

The calculation results show that the non-directional protection will work correctly not at all connections (Feeder-2 and Feeder-8). In this case, the setpoint values were coarsened by the condition for choosing the total capacitive current. Therefore, if at least one connection is disconnected, then the protection performance will be an order of magnitude worse. In this case, the protection is ineffective even under normal network operation.

If it is impossible to form a reliable non-directional protection, it is necessary to consider a directional type of protection against single-phase earth faults (current non-directional zero sequence protection).

Calculation of setpoints for directional zero-sequence protection using the example of the Feeder-1 connection is presented as follows.

The operating current is $2.95 \mathrm{~A}$. The total capacitive current is taken equal to the capacitive component of the current of single-phase earth faults with two working connections on the first bus section and three connections on the second bus section.

Tuning away from the own capacitive current of the connection is not required, since the failure of an external single-phase earth fault is provided by the phase characteristic.

The tripping current of the protection relay is $0.05 \mathrm{~A}$.

The results of calculating the setpoints of nondirectional zero-sequence protection are presented in Table 4.

Table 4. The results of calculating the setpoints of nondirectional zero-sequence protection.

\begin{tabular}{|c|c|c|c|c|}
\hline $\begin{array}{c}\text { No. } \\
\text { of } \\
\text { unit }\end{array}$ & Connection & $\begin{array}{c}\text { Protection } \\
\text { operation } \\
\text { current, A }\end{array}$ & $\begin{array}{c}\text { Relay } \\
\text { operation } \\
\text { current, A }\end{array}$ & $\begin{array}{c}\text { Operation } \\
\text { time, s }\end{array}$ \\
\hline 1 & Feeder -1 & 2.95 & 0.05 & 0.3 \\
\hline 2 & Feeder -2 & 1.78 & 0.03 & 0.3 \\
\hline 4 & Feeder -4 & 4.42 & 0.07 & 0.3 \\
\hline 5 & Feeder -5 & 5.28 & 0.09 & 0.3 \\
\hline 6 & Feeder -6 & 4.92 & 0.08 & 0.3 \\
\hline 7 & Feeder -7 & 4.92 & 0.08 & 0.3 \\
\hline 8 & Feeder -8 & 3.59 & 0.06 & 0.3 \\
\hline 10 & Feeder -10 & 3.93 & 0.07 & 0.3 \\
\hline
\end{tabular}

Regardless the calculation results, it is important to understand that there was no tuning away from the total unbalance current, since it is currently not possible to calculate its exact value. This problem is the only drawback of directional protection.
This paper provides a sequence of actions necessary for the correct operation of directional zero sequence protection. To begin with, in the connection protection terminals, the calculated setpoints from Table 4 are set. If the established settings do not ensure the protection performance, then they must be gradually coarsened during operation until the protection becomes operational.

The proposed principle for selecting directional protection setpoints is shown in Figure 4.

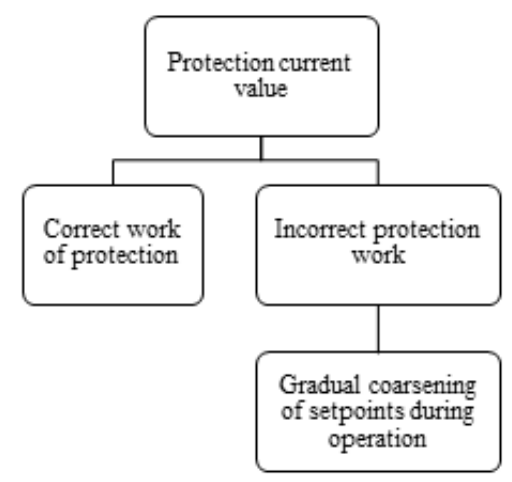

Fig. 4. Sequential setpoint selection of current directional zero sequence protection.

The main advantage of decentralized protection terminals is that there is no need to install additional equipment, since bay protection terminals are installed regardless of the type of zero sequence protection. With correct setpoints, directional protection will work selectively, regardless of the network operating mode. Nevertheless, there are limits for the protection operation current not only for the total unbalance current, but also for the relay operation current, since it must be at least $0.01 \mathrm{~A}$.

\section{Conclusions}

This work presents modeling of a $10 \mathrm{kV}$ electrical network of a 110/35/10 kV substation with insulated neutral. Centralized and non-centralized protection against earth faults with action on signal and trip are investigated. The setpoints of directional and nondirectional zero sequence protections were calculated. For protection against single-phase earth faults, directional and non-directional protections with relative and absolute metering were used.

For a network with an insulated neutral, centralized undirected protection with relative metering turned out to be the most effective. However, there are network operating modes in which they become inoperative. Conventional non-directional zero sequence protection based on the bay terminal has proven to be completely ineffective, even when the network operation is most favorable for relay protection. Directional zero sequence protection, formed on the same terminal, has sufficient sensitivity, however, due to the impossibility of correctly calculating the total unbalance current, it is not reliable. In conclusion, It should be noted that it is advisable to exclude a network with an insulated neutral from 
operation. This type of earthing has the only advantage that cannot be considered unambiguous, since there can appear a second short circuit at another connection due to overvoltages.

\section{References}

1. X. Song, F. Gao, Z. Chen, W. Liu A negative selection algorithm-based identification framework for distribution network faults with high resistance. IEEE Access, 7, 109363-109374. (2019). https://doi.org/10.1109/ACCESS.2019.2933566.

2. D.P.S. Gomes, C. Ozansoy, A. Ulhaq Highsensitivity vegetation high impedance fault detection based on signal's high-frequency contents. IEEE Transactions on Power Delivery, 33(3), 1398-1407. (2018). https://doi.org/10.1109/TPWRD.2018.2791986.

3. Q. Cui, K. El-Arroudi, Y. Weng, A feature selection method for high impedance fault detection. IEEE Transactions on Power Delivery, 34(3), 1203-1215 (2019). https://doi.org/10.1109/TPWRD.2019.2901634.

4. S. Chakraborty, \& Das, S. Application of smart meters in high impedance fault detection on distribution systems. IEEE Transactions on Smart Grid, 10(3), 3465-3473 (2018). https://doi.org/10.1109/TSG.2018.2828414.

5. V. Vukolov, M. Obalin, A. Petrov Improvement of algorithms for voltage circuits fault detection in relay protection terminal of $6-35 \mathrm{kV}$ electrical networks. E3S Web of Conferences 139, 01061(2019). https://doi.org/10.1051/e3sconf/201913901061.

6. P.M. Mircea, G. Buzatu, I. Mircea, I. Marin, D. Ursu and F. Bocai, Overvoltages Evaluation in Electrical Networks with Insulated Neutral Analytical Calculation and Experimental Tests 2017 European Conference on Electrical Engineering and Computer Science (EECS), Bern, 2017, pp. 380385, doi: 10.1109/EECS.2017.77.

7. P.M. Mircea, M. Ciontu, I. Marin, I. Mircea, D. Rusinaru, G.C. Buzatu, F. Popescu, The influence of neutral treatment system type on the efficiency / performance of the MV networks, Applied and Theoretical Electricity (ICATE) 2018 International Conference on, pp. 1-6, 2018.

8. A.N. Alyunov, O.S. Vyatkina, I.G. Akhmetova, B.E. Umirzakov, D.A. Tashmukhamedova Creation of a mathematical model to identify short circuits in power lines. E3S Web of Conferences 124, 02019 (2019) https://doi.org/10.1051/e3sconf/201912402019.

9. Smagulova, K. \& Iskakov, Ualikhan. (2017). Simulation Modes Of Relay Protection Devices In Networks With Insulated Neutral. EAI Endorsed Transactions on Energy Web. 4. 153468. 10.4108/eai.13-12-2017.153468.
10. P.M. Mircea, M. Ciontu, I. Mircea, C. Buzatu, D. Rusinaru, D. Ursu, I. Marin, E. Butoarca, Analyzing the Efficiency of Neutral Treatment with Resistor and Shunt Circuit Breaker in a MV Distribution Substation, Control Artificial Intelligence Robotics \& Optimization (ICCAIRO) 2018 International Conference on, pp. 223-228, 2018.

11. P. M. Mircea, D. Ursu, I. Marin, I. Mircea and G. C. Buzatu, Analysis of overvoltages occurring at grounding faults in a medium voltage network depending on the neutral treatment. The 23 th Conference of Energy Engineering CIE, 2017.

12. Pinchukov, P. Modelling of Dynamic Compensation for Insulated Neutral Power Network. IOP Conference Series: Earth and Environmental Science. 272. 022070. (2019). doi: 10.1088/1755$1315 / 272 / 2 / 022070$. 